\title{
Blood flow in stenosed arteries using two way, fluid-structural interaction
}

\author{
$\begin{array}{lll}\text { C. X. Chen } & \text { Y. Ding } & \text { J. A. Gear } \\ & \end{array}$
}

(Received 30 December 2009; revised 14 August 2010)

\begin{abstract}
Blood flow in a stenosed artery was simulated using the two way, fluid-structural interactions in ANSYS - a commercial finite element and finite volume software widely used in industry. Several stenosis models were investigated using a pulsatile flow condition. In these models the form of the stenosis geometry was either axisymmetric or axisasymmetric. The blood was modelled both as a non-Newtonian, power law fluid, and as the Casson model. The artery tissue was assumed to be a linear elastic material for the axis-symmetric stenosis model and nonlinear material for the axis-asymmetric stenosis models. A fibrous cap and lipid pool were also added into the stenosis geometry of the axis-asymmetric models to account for complexities of the physiology. The effect of the stenosis severity on arteries was investigated by examining the blood flow velocity, pressure and wall shear stress at multiple locations.
\end{abstract}

http://anziamj . austms.org.au/ojs/index.php/ANZIAMJ/article/view/2575 gives this article, (C) Austral. Mathematical Soc. 2010. Published August 25, 2010. ISSN 1446-8735. (Print two pages per sheet of paper.) Copies of this article must not be made otherwise available on the internet; instead link directly to this URL for this article. 


\section{Contents}

1 Introduction

C587

2 Methods and settings

C589

3 Results and discussion

C590

4 Conclusion

C608

References

C609

\section{Introduction}

Though mathematical modelling of blood flow through a narrow arterial tube has been a classical problem dating back to the 1840's after the pioneer experimental work conducted by Jean Louis Marie Poiseuille [8], numerical simulations on atherosclerosis only started to thrive in 1990s due to the rapid development in computer technology. Chakravarty et al. [3] treated blood as a Newtonian fluid. Ang and Mazumdar [1] presented a 3D numerical simulation of flow through an asymmetric arterial stenosis. Four severities of stenosis were used. The results indicated that the stenosed side of the wall had much higher shear stress when compared to the healthy side of the wall. Pincombe and Mazumdar [7] studied the effects of post stenotic dilations in stenosed arteries. Using the Bingham model, the resistance to blood flow increased as the stenosis severity increased, and decreased as the dilation increased. Overall, the Bingham model was found to be more suitable for small arteries due to its decreased response to changes in arterial radius when compared to the Newtonian fluid model. Among the several popular non-Newtonian fluid models, Siddiqui et al. [9, 10] proved that the Casson model was particularly accurate when modelling blood flow. Their results showed the yield stress grew proportionally to the plague core radius. 
Blood flow through a stenosed artery results in the elastic deformation of the artery. In return the deformation affects the flow behaviour. Hence, numerical simulation using fluid-structural interactions is becoming the preferred solution method. Lee and $\mathrm{Xu}$ [5] studied a Newtonian blood flow pattern through a stenosed artery vessel and the behavior of the artery wall using one way fluid-structure interactions (FSI) - an early stage of the numerical simulation technique. Chan et al. [2] adopted Lee and Xu's stenosis model to study the non-Newtonian blood flow pattern using one way FSI technique. Their results showed that the artery wall deformed the most at the down stream end of the stenosis, and the pressure in the flow experienced a constant drop in the pre-stenotic region followed by a sharp fall when passing the stenosis and an increase in the post-stenotic region. Chan et al. also noted a high stress concentration at the shoulder of the stenosis. The numerical simulation results from Chan et al. [2] and Lee and Xu [5] provided insightful information on the wall shear stress (WSS) when blood flow and artery vessel interact. However, they assumed that both the stenosis and artery wall possessed the same material property. Such an assumption, although widely used, ignored the physiological structure of a plague, which has a fibrous cap and lipid pool with different material properties from the artery wall.

Tang et al. $[11,12]$ investigated plaque rupture due to the influence of the artery wall on blood flow. In order to resemble the physiological condition of a plague, they used a thick-wall model, in which the material property of the stenosis region was different to that of the artery wall. Another development in the field has been the use of MRI imaging. Tang et al. $[13,14]$ also made valuable contributions in this area. Their studies showed that the artery wall material had to be chosen carefully in order to obtain accurate results; and that plaque progression was dependent on the stress and strain of the artery wall and the wss of the blood flow.

Due to the complexities of the numerical methods involved for a real life simulation of blood flow through a stenosed artery, little research has been done incorporating the FSI simulation along with: 
1. non-Newtonian blood rheology;

2. axis-asymmetric stenosed artery geometries; and

3. a stenosis model that resembles the physiological structure of a plague. This is the objective of our research. The stenosed artery model used in this study includes all the three modelling aspects listed above; that is, non-Newtonian fluid models for blood, 3D axis-asymmetric stenosed artery geometries, and the atherosclerotic plague constructed by a fibrous cap and lipid pool with multiple material regions, each region has a distinct material property and localised stiffness. The model includes two levels of severity, $45 \%$ and $78 \%$ flow path area reductions, and is solved, respectively, using two way fluid-structural interactions (FSI) in order to determine the critical value of the wss for plaque rupture. Velocity profiles are also monitored to determine the size of flow recirculation zones.

\section{Methods and settings}

The two way FSI solution concerns a pulsatile blood flow of period tp $=0.345 \mathrm{~s}$ through an elastic artery vessel with a localised path restriction of either a $45 \%$ or $78 \%$ area reduction. 69 time steps are used. At each time step, the blood flow, being viscous and incompressible, is governed by the continuity and Navier-Stokes equations and solved in ANSYS-CFX using the finite volume method. The resolved fluid pressure at the interface of the blood and artery wall is then applied as a load on the wall to solve for the deformation of the artery in ANSYS-Mechanical using the finite element method. The displacement obtained in the structural analysis is transferred back to ANSYS-CFX to adjust the fluid domain and update the blood flow analysis. This procedure continues until overall equilibrium is reached between the CFD solution and structural solution.

Several plaque geometries were used. The first model was a 3D carotid artery 
with an axisymmetric stenosis. This model was constructed to validate the model settings for our two way FSI simulations in 3D. The cross-section of the model along its axis was similar to the 2D model used by Chan et al. [2], with smoother edges and corners of the plaque for a more realistic representation. The 2D model of Chan et al. was solved using one way FSI method, whereas our 3D validation model was solved using two way FSI. In order to compare results, the blood rheology was described by the Power Law model as by Chan et al. [4]. Young's modulus of the artery wall was chosen as $10 \mathrm{MPa}$. After the model settings were confirmed, the 3D axis-asymmetric models of an elliptical form of $45 \%$ and $78 \%$ stenosis severities were calculated, with a neo-Hookean model describing the behaviour of the arterial wall and a Casson model for the blood rheology. The stenotic models were then further modified to include multiple material regions: a lipid pool and a fibrous cap with $0.3 \mathrm{~mm}$ thickness. The fibrous cap has the same material property as the artery wall, whereas the lipid pool uses a Young's modulus of $100 \mathrm{kPa}$. For the $78 \%$ axis-asymmetric stenosis models, it was reasonable to assume that the diseased tissue would harden over time. Therefore a localised stiffness was applied for the stenosis region [6], which was four times stiffer than the $45 \%$ severity models.

\section{Results and discussion}

The model validation was done by comparing the wall shear stress (Wss) resulting from the Power Law blood flow model to that of Chan et al. [2] at the normalized time step $t / t p=0.25$ (Figure 1). Velocities were compared at the normalized post stenotic position $Z^{\prime}=4.3$, where we define $Z^{\prime}=Z_{1} / D$ with $Z_{1}$ being the axial distance from the center of the stenosis $(Z=0.016 \mathrm{~m})$ towards downstream and $\mathrm{D}$ the inner diameter of the artery. As shown in Figure 1, the maximum wsS occurs at about $0.0162 \mathrm{~m}$ from the inlet for the time step $t / t p=0.25$. This is supported by the results of Chan et al. The results of Chan et al. only provide wss values from $0.01 \mathrm{~m}$ to $0.05 \mathrm{~m}$, whereas 

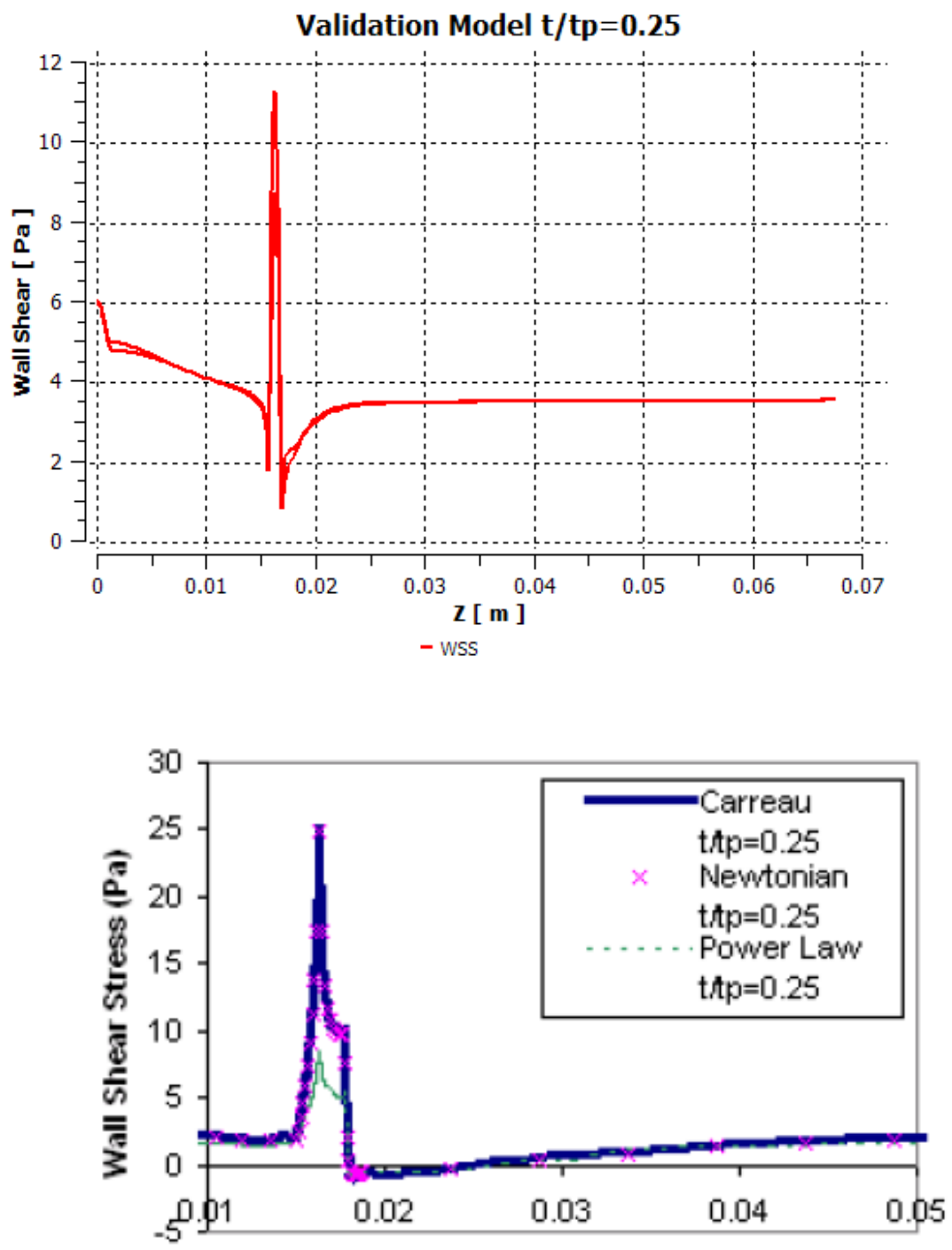

Axial Distance (m)

Figure 1: WSS of Power Law flow at $t / t p=0.25$ compared with that of Power Law flow of Chan et al. [2] below. 
the results of our 3D models show the WSS variations of the entire artery from the inlet to outlet. Therefore, the initial wss decline was not revealed on all the results of Chan et al. The peak wss value is slightly higher than the result from the Power Law flow of Chan et al. This variation is expected as the stenosis shape is slightly different.

Figure 2 shows the WSS results for the $45 \%$ axis-asymmetric stenosis model with uniform wall material properties. Unlike the validation model, at each time step there are two peaks in the asymmetric model. The curve with the higher peak represents wss from the stenosed side of the wall; while the one with the lower peak represents wss from the healthy side of the wall. The maximal higher peak WSs is $2.4 \mathrm{~Pa}$ at $\mathrm{t} / \mathrm{t} \mathrm{p}=0.25$ (systolic phase) and the minimal higher peak WSS is $0.75 \mathrm{~Pa}$ at $\mathrm{t} / \mathrm{tp}=0.75$ (diastolic phase). As shown in Figure 3, the pressure on the artery wall varies mostly in a linear fashion. However, the pressure drop difference at the stenosis region is noticeable, around $100 \mathrm{~Pa}$ for the diastolic phase and $200 \mathrm{~Pa}$ at the systolic phase. Pressure of all time intervals converges to the specified outlet pressure, which again suggests that the model works properly. Velocity at the post stenotic region was also investigated. Figure 4(a) shows that the velocity variation at $Z^{\prime}=4.3$ lost its sinusoidal profile towards the end of the time period. This suggests that the artery blockage forces blood to travel at a higher speed at the stenosis contraction. The velocity vector plot in Figure 4(b) shows a maximum velocity of $60 \mathrm{~cm} \mathrm{~s}^{-1}$ with a large flow recirculation immediately after the stenosis.

Figure 5 provides the Wss resulting from the multi-material stenosis model. The maximum Wss is $3 \mathrm{~Pa}$ and is observed in the systolic phase $\left(t / t_{p}=0.25\right)$ of Figure 5(b). Comparing to the Wss of the uniform wall material $45 \%$ stenosis model (Figure 2), the result of the multi-material stenosis model is more realistic as there is no sharp rise in the wss on the healthy side of the wall and less fluctuation of Wss in the post stenotic phase $\left(t / t_{p}=0.75\right)$. The multi-material stenosis model is also confirmed by the pressure results (Figure 6). Results at both time steps show less pressure fluctuation in the post stenotic region. The pressure variation at the stenotic contraction region 



FigURE 2: WSS variations along the $45 \%$ axis-asymmetric stenosed artery with uniform wall material properties at two different time steps. 

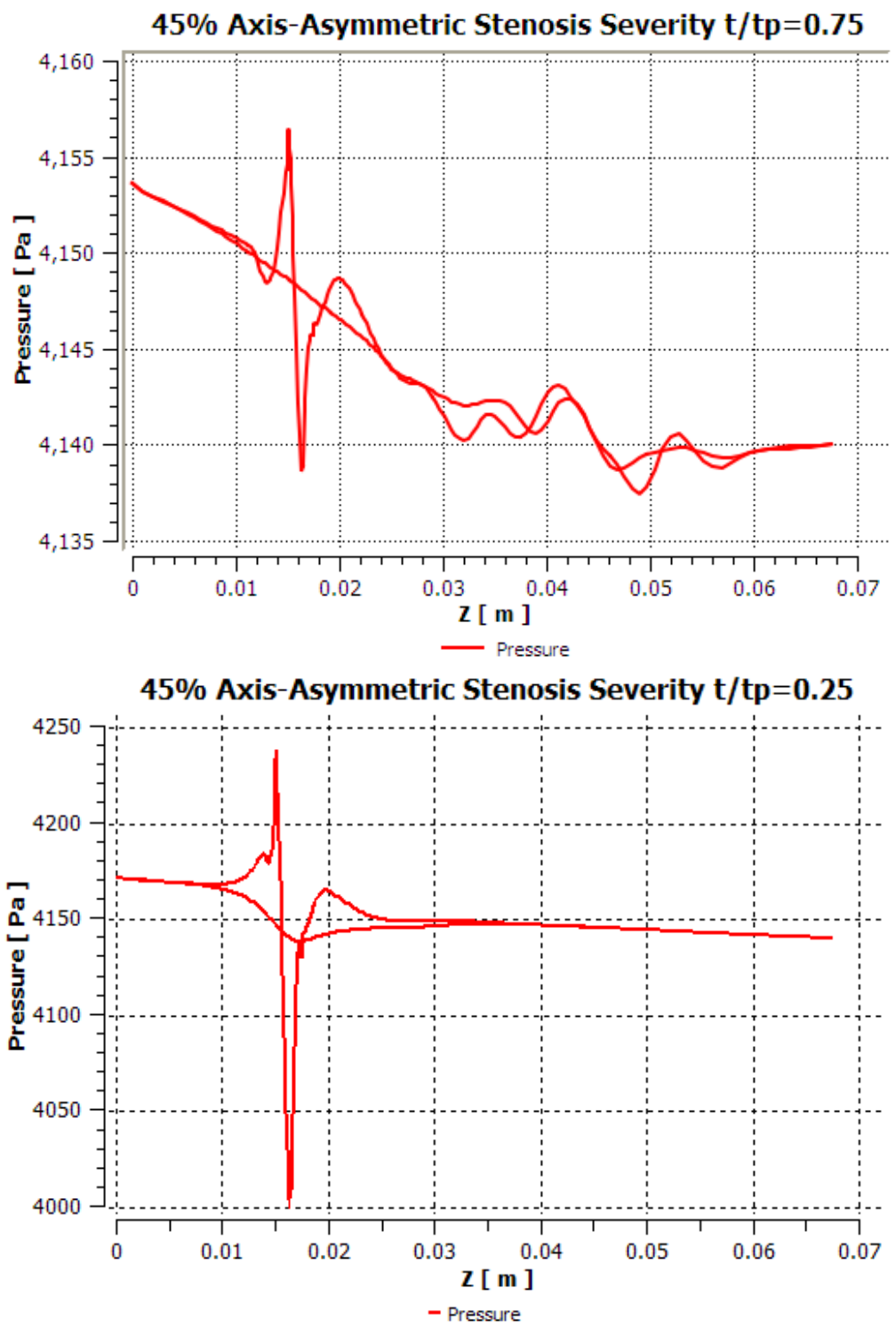

Figure 3: Pressure plots for the $45 \%$ axis-asymmetric stenosed artery with uniform wall material properties at two different time steps. 




- Velocity

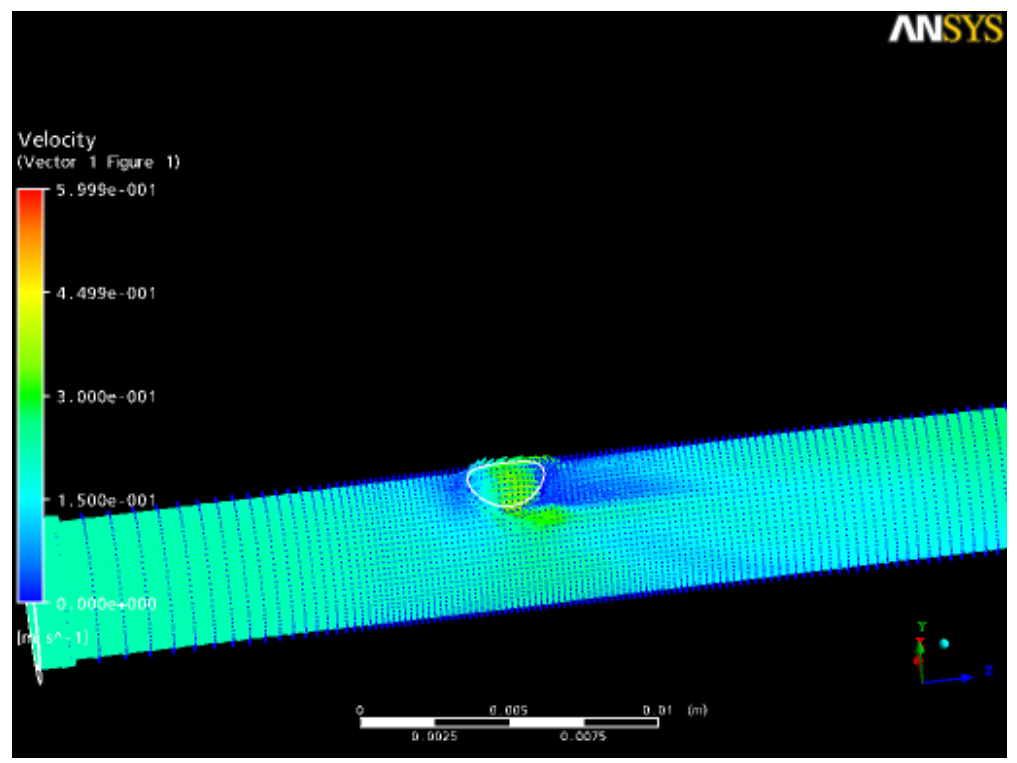

FiguRE 4: Velocity plots for 45\% axis-asymmetric stenosed artery with uniform wall material properties: (a) velocity magnitude versus time at $\mathrm{Z}^{\prime}=4.3$; (b) velocity vector plot at time $\mathrm{t}=0.345 \mathrm{~s}$. 
Fibrous Cap Stenosis $\mathbf{t} / \mathbf{t p}=\mathbf{0 . 7 5}$

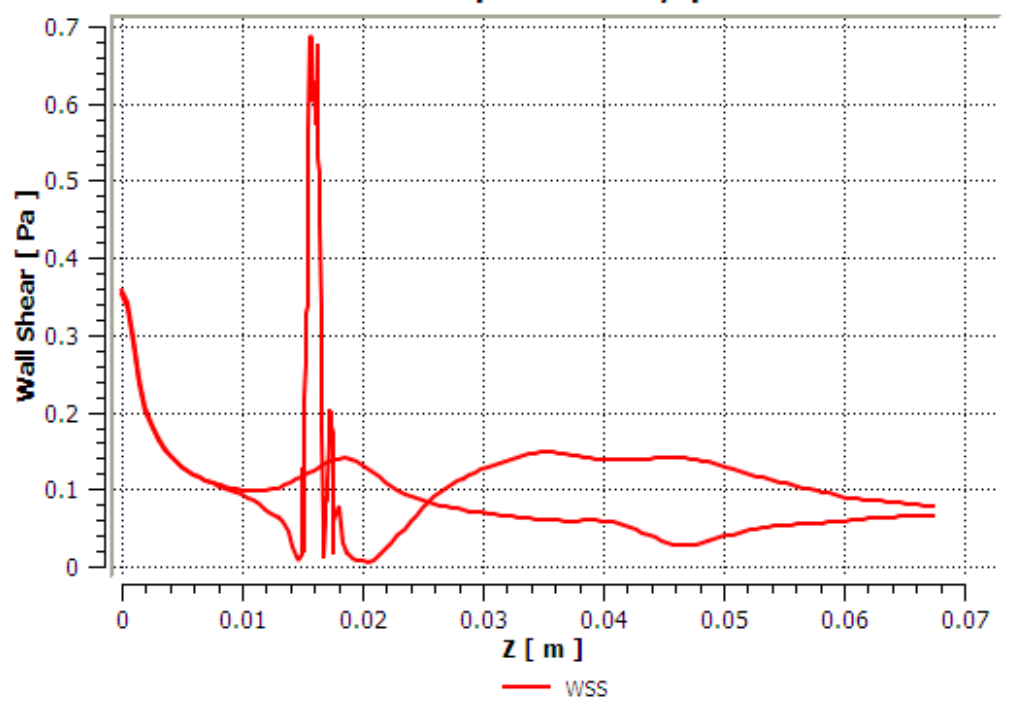

Fibrous Cap Stenosis $\mathrm{t} / \mathrm{tp}=\mathbf{0 . 2 5}$

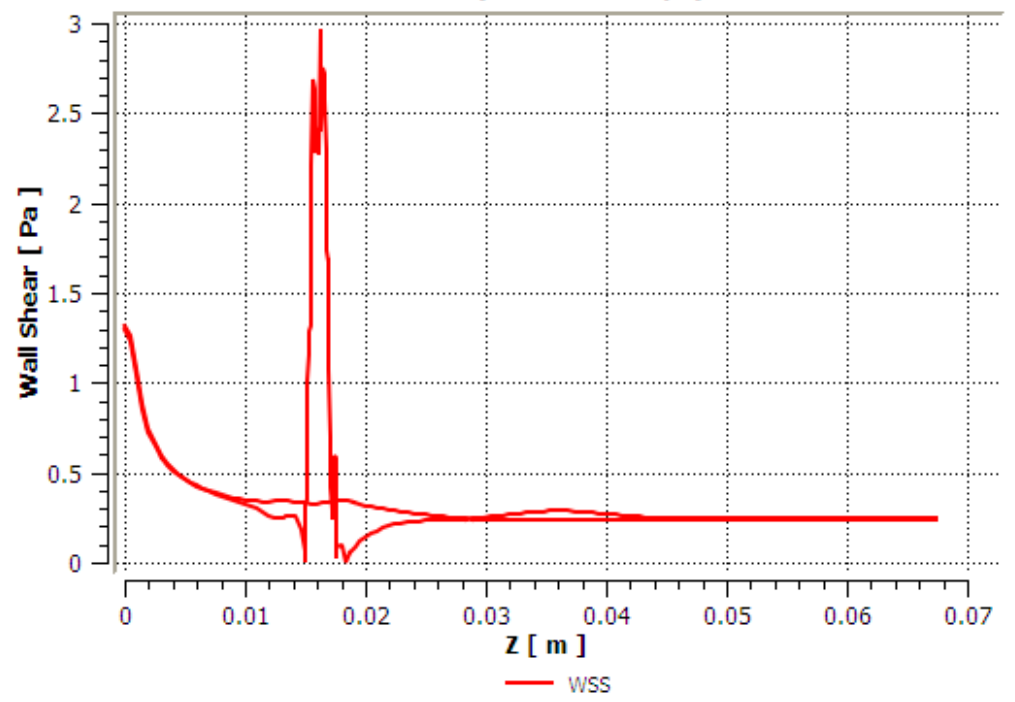

FigURE 5: WSS plots for $45 \%$ axis-asymmetric stenosed artery with multimaterial properties at two different time steps. 
is similar to the uniform material model, at all time steps. The pressure trend is almost linear in both pre and post stenotic regions, with no major fluctuation at the stenotic contraction. Furthermore, the velocity magnitude in the post stenotic region varies with time in a smooth sinusoidal profile as shown in Figure 7(a). There is no velocity distortion throughout the time period. For a $45 \%$ stenosis, that is a relatively mild case of atherosclerosis, velocity distortion is not expected. Therefore, the multi-material stenosis model is proven to be more accurate, hence the preferred model. The velocity vector plot (Figure 7(b)) also shows less flow recirculation in the post stenotic region. This is due to the softer material properties of the lipid pool portion of the stenosis, which makes the stenosis more flexible and less resistive to the blood flow.

The $78 \%$ severity stenosis is considered as the most severe form of the disease. Though its maximum wss values for the uniform material stenosis model (Figure 8) are similar to that of the $45 \%$ severity model, there is a significant increase in the Wss values in the stenotic contraction and post stenotic regions. This increase is due to the high severity of the stenosis and much narrower passageway for blood flow, creating the high shear stress on both surfaces of the wall. Pressure drops (Figure 9) are much greater when compared to that of the $45 \%$ severity models. This is expected, as the stenosis severity is higher, the pressure drop at the stenotic contraction would be greater. The maximum pressure drop is $350 \mathrm{~Pa}$ at $\mathrm{t} / \mathrm{t} \mathrm{p}=0.25$, the lowest pressure drop is $26 \mathrm{~Pa}$ at $\mathrm{t} / \mathrm{t} p=0.75$. The pressure trends are very similar when compared to the $45 \%$ severity models at all other time steps in the pre and post stenotic regions. Pressure fluctuation is only observed at the diastolic phase for both $45 \%$ and $78 \%$ severities.

Velocity profiles at the post stenotic point indicates a major distortion of the sinusoidal profile, shown in Figure 10. The vector plot shows a maximum velocity of around $74 \mathrm{~cm} \mathrm{~s}^{-1}$ at the stenotic region and a large zero velocity area before the stenosis. This high velocity and the narrowed passageway would be a main contributing factor to the velocity profile distortion. 
Fibrous Cap Stenosis $\mathrm{t} / \mathrm{tp}=\mathbf{0 . 7 5}$

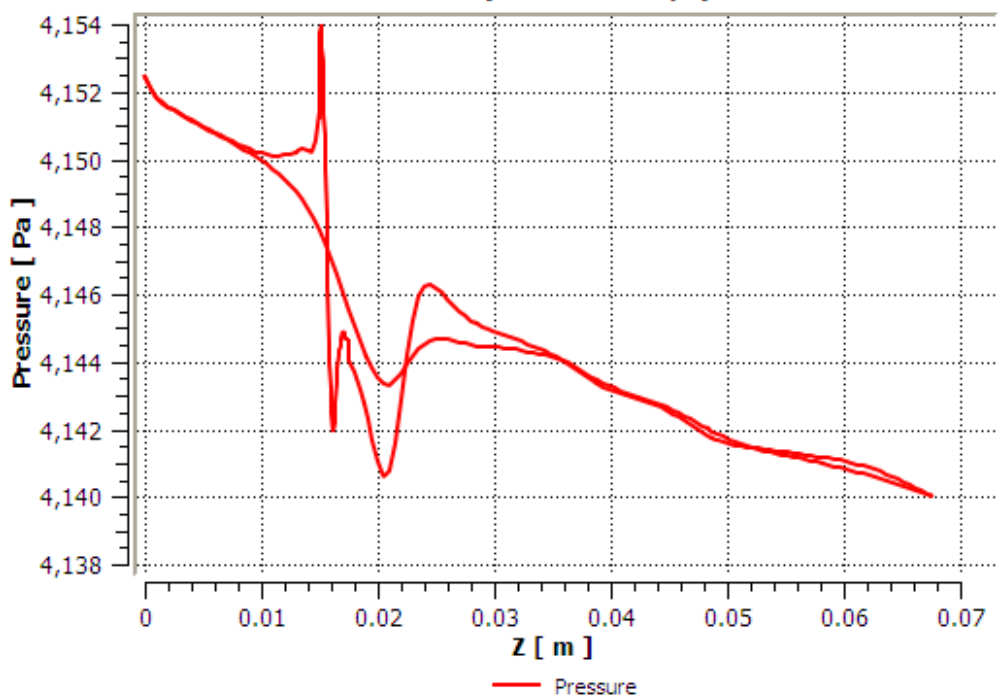

Fibrous Cap Stenosis $\mathrm{t} / \mathrm{tp}=\mathbf{0 . 2 5}$

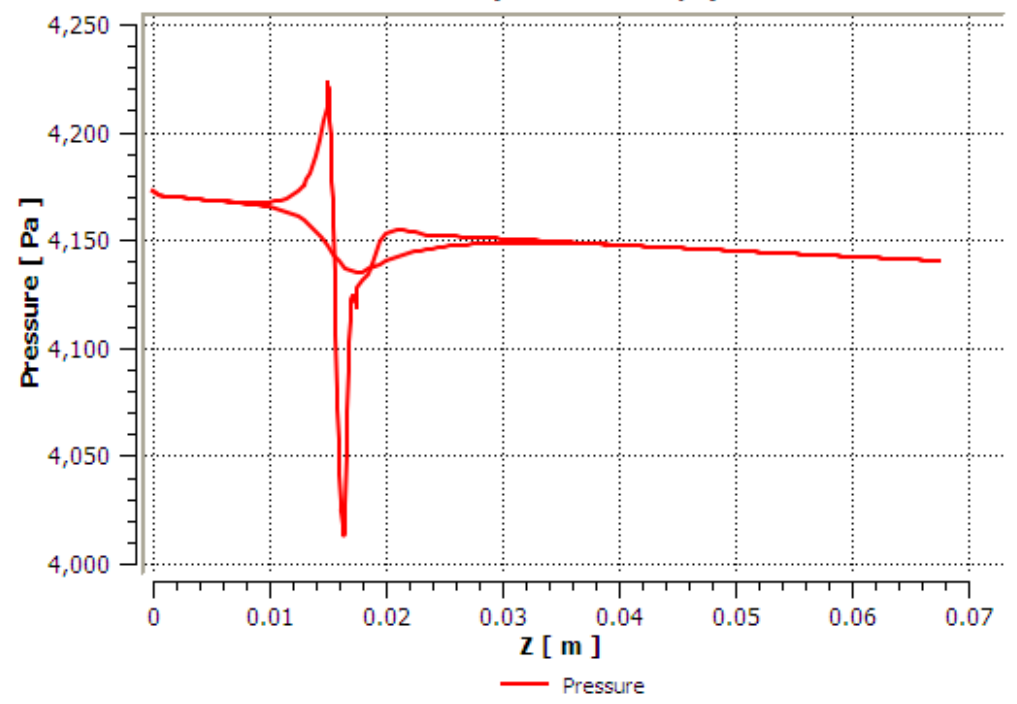

Figure 6: Pressure plots for $45 \%$ axis-asymmetric stenosed artery with multi-material properties at two different time steps. 
Fibrous Cap Stenosis Z'=4.3

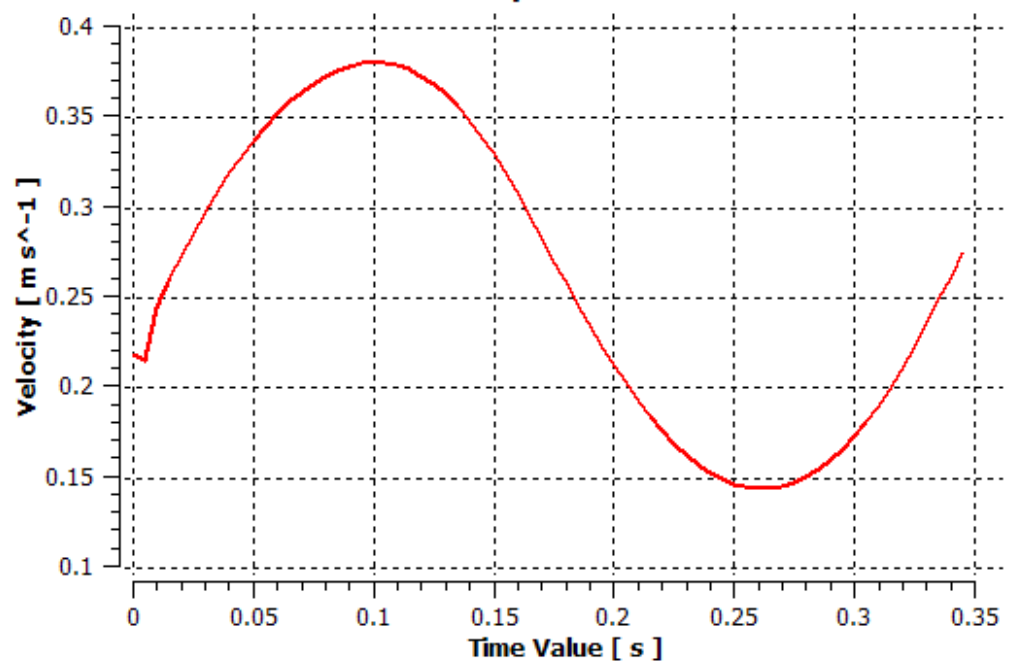

- Velocity

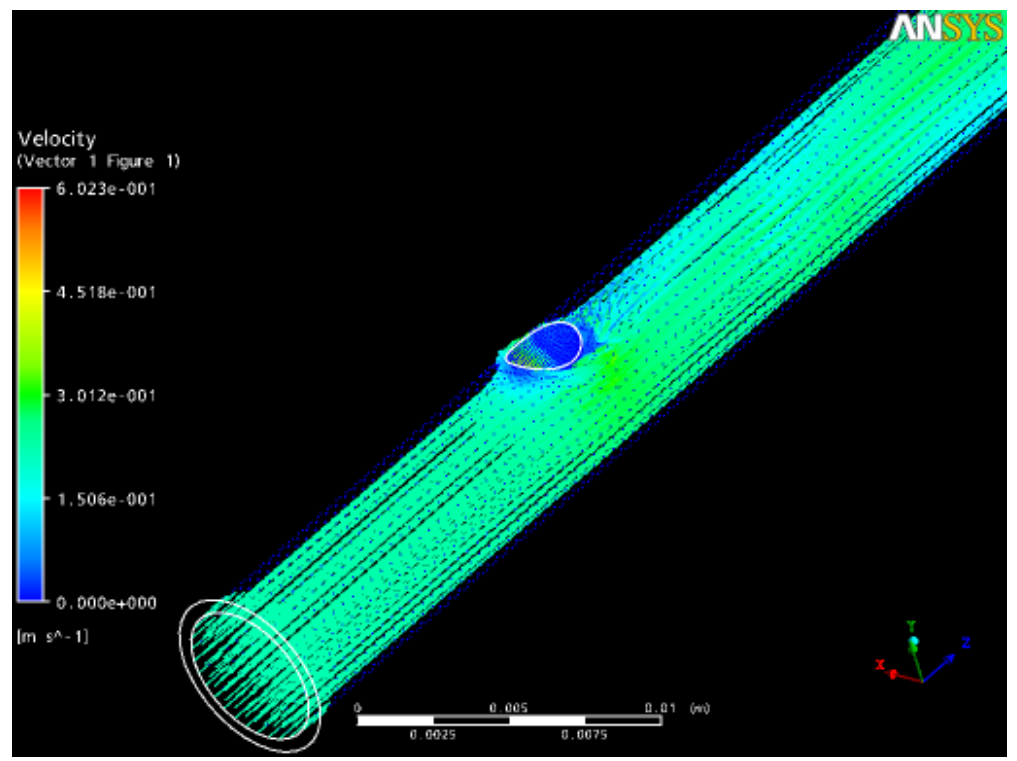

FiguRE 7: Velocity plots for $45 \%$ axis-asymmetric stenosed artery with multi-material properties: (a) velocity magnitude versus time at $Z^{\prime}=4.3$; (b) velocity vector plot at time $t=0.345 \mathrm{~s}$. 

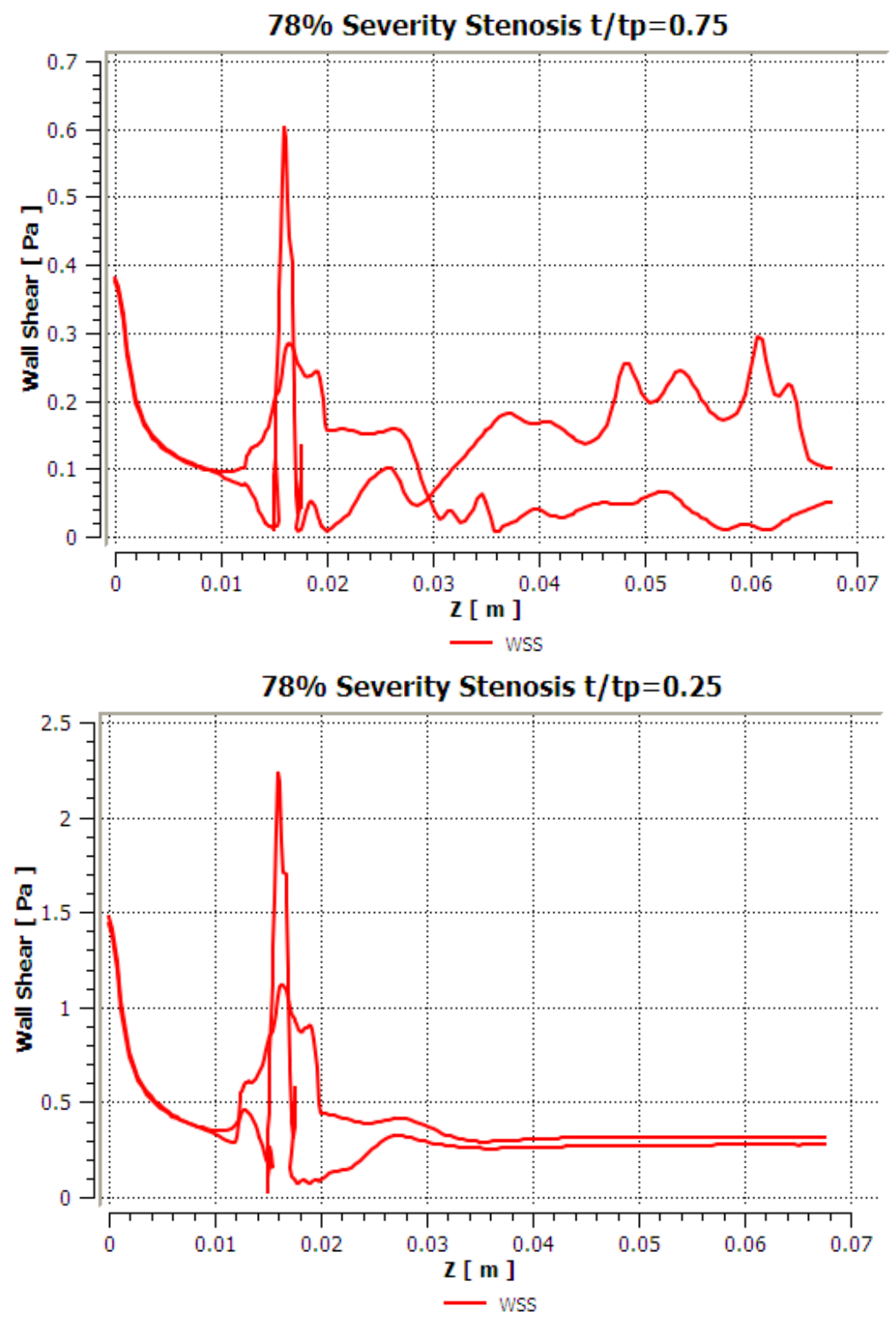

FIGURE 8: WSS plots for $78 \%$ axis-asymmetric stenosed artery with uniform wall material properties at two different time steps. 

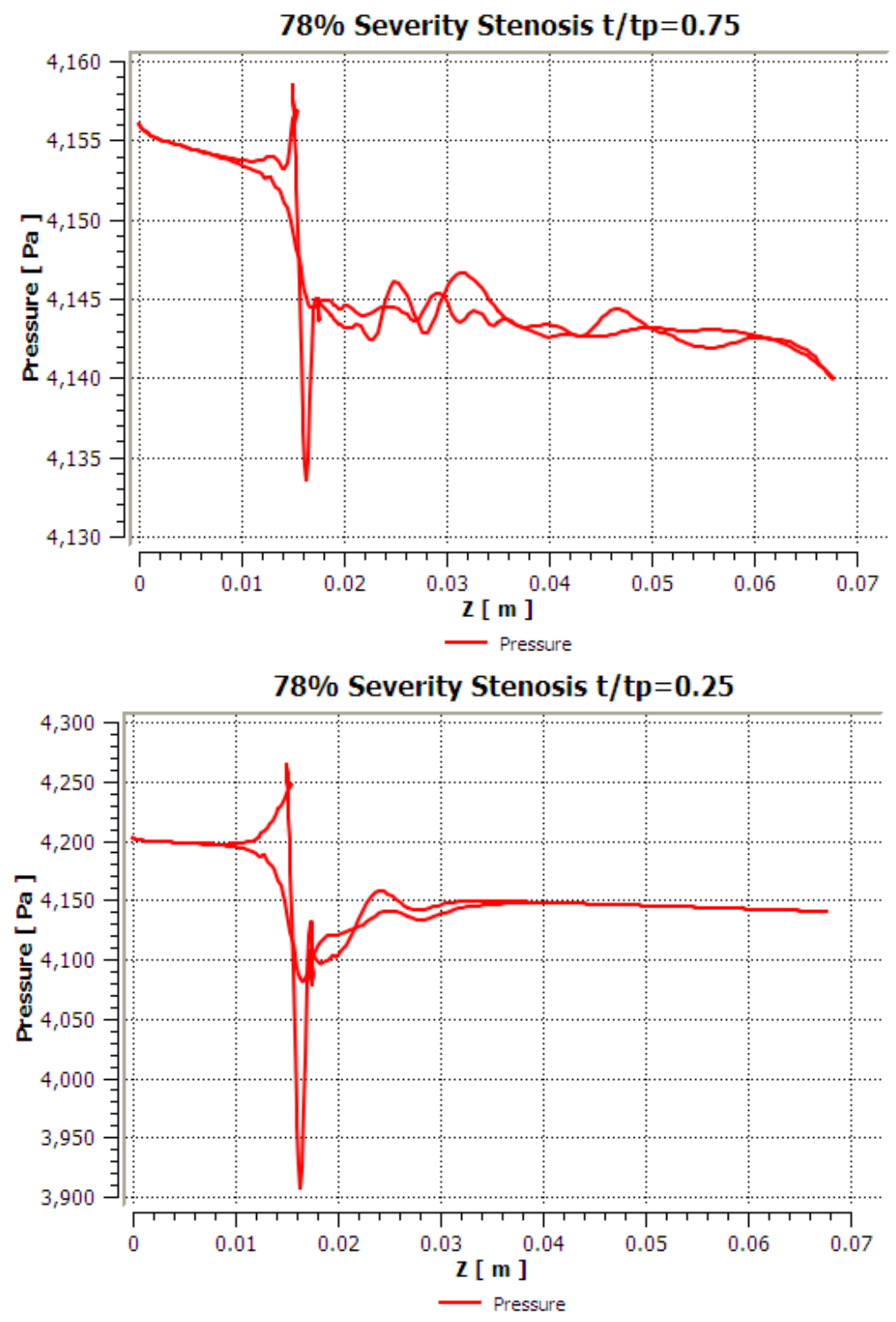

Figure 9: Pressure plots for $78 \%$ axis-asymmetric stenosed artery with uniform wall material properties at two different time steps. 




- Velocity

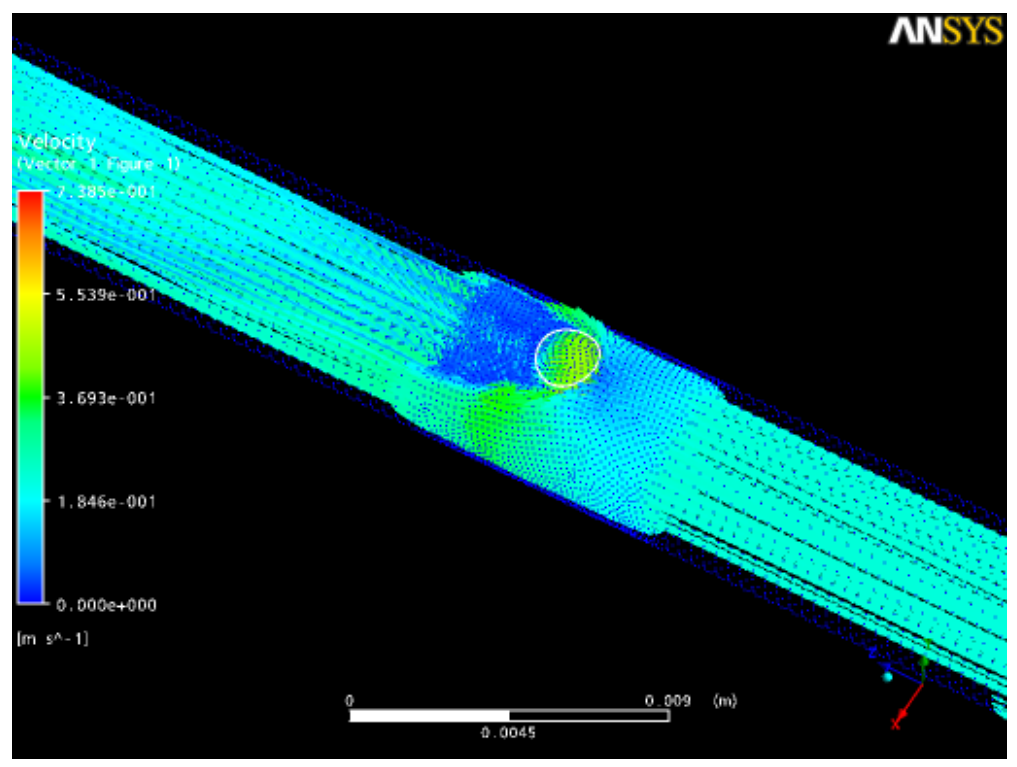

FiguRE 10: Velocity plots for $78 \%$ axis-asymmetric stenosed artery with uniform wall material properties. 

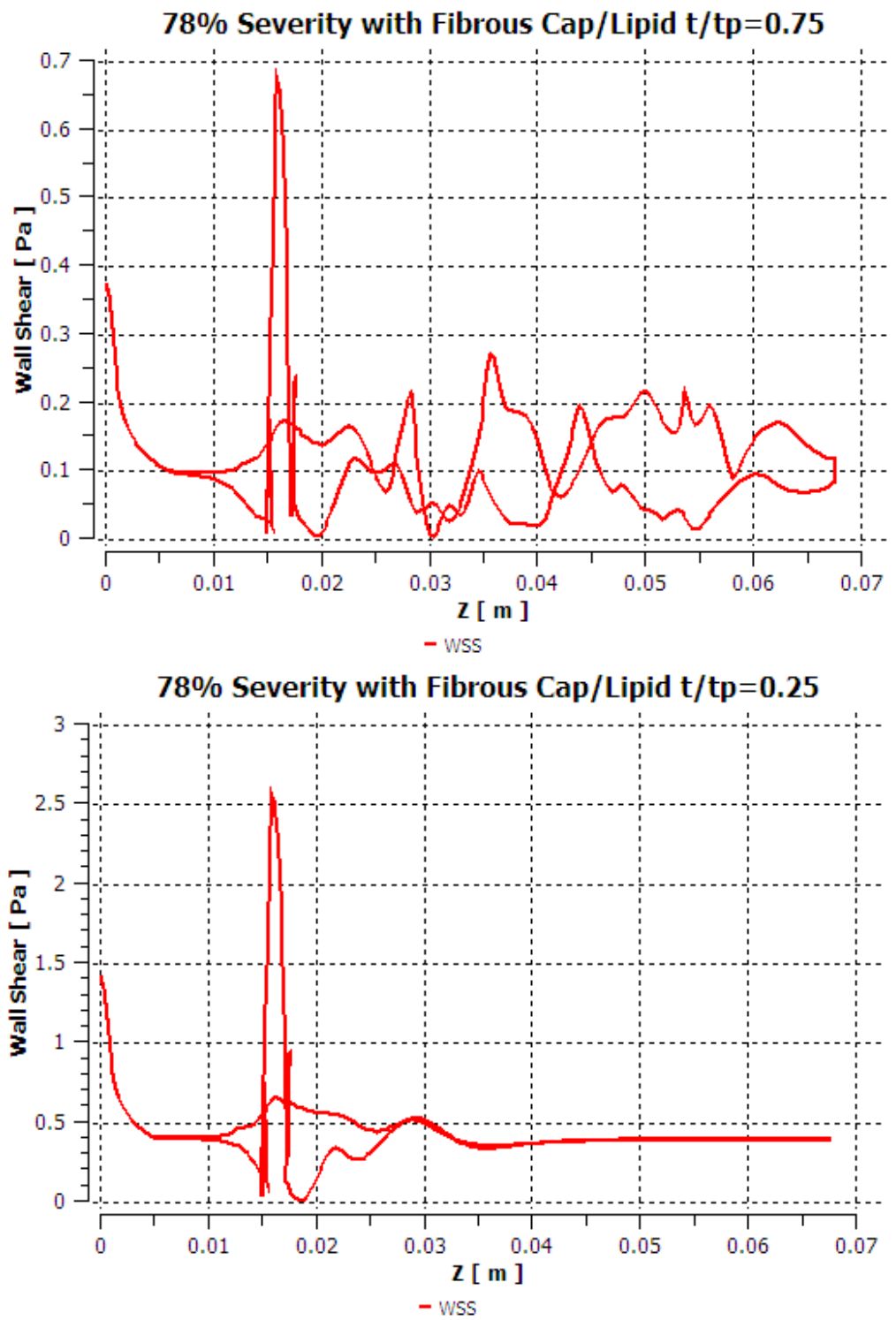

FigURE 11: WSS plots for $78 \%$ axis-asymmetric stenosed artery with multimaterial properties at two different time steps. 

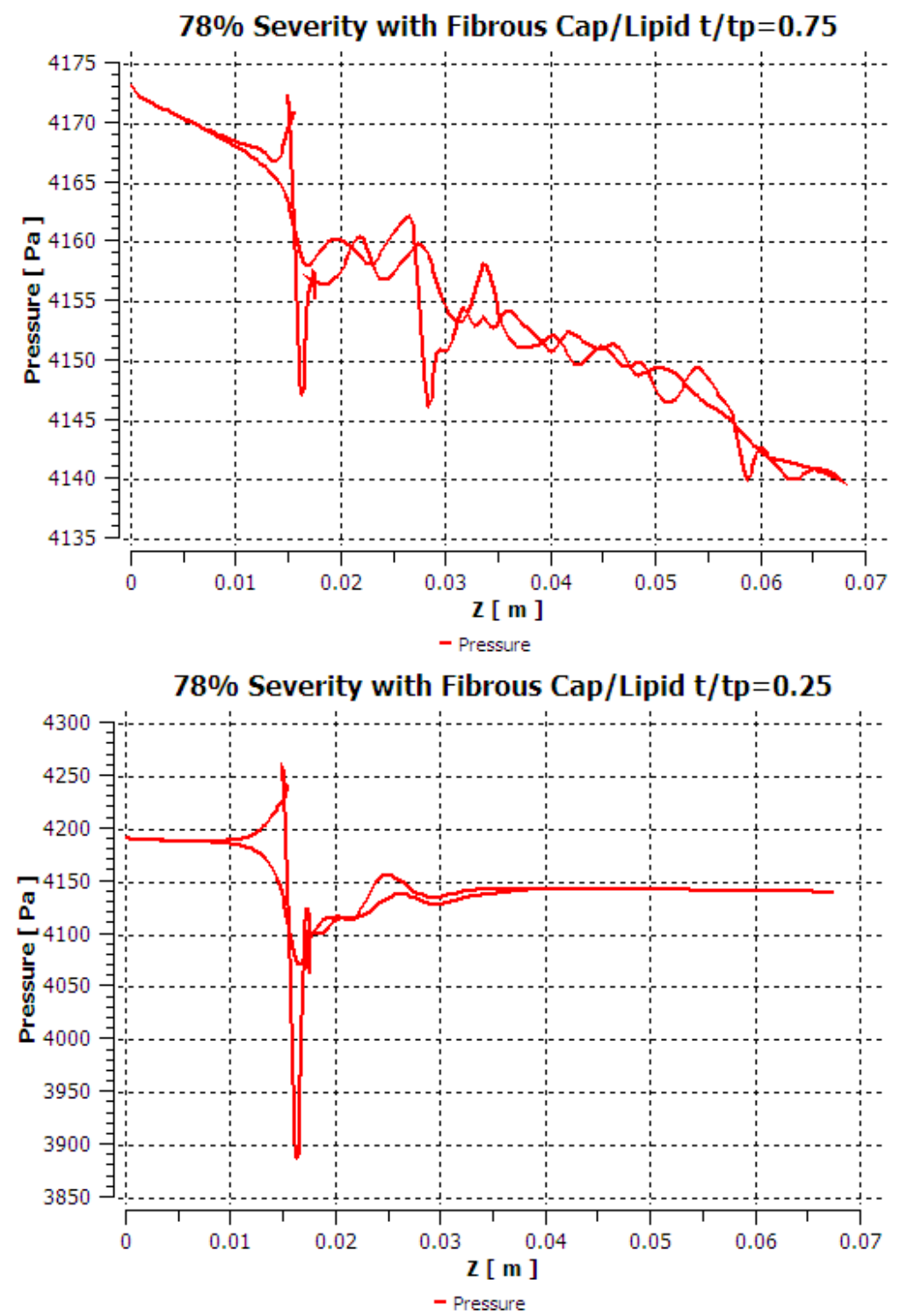

Figure 12: Pressure plots for $78 \%$ axis-asymmetric stenosed artery with multi-material properties at two different time steps. 


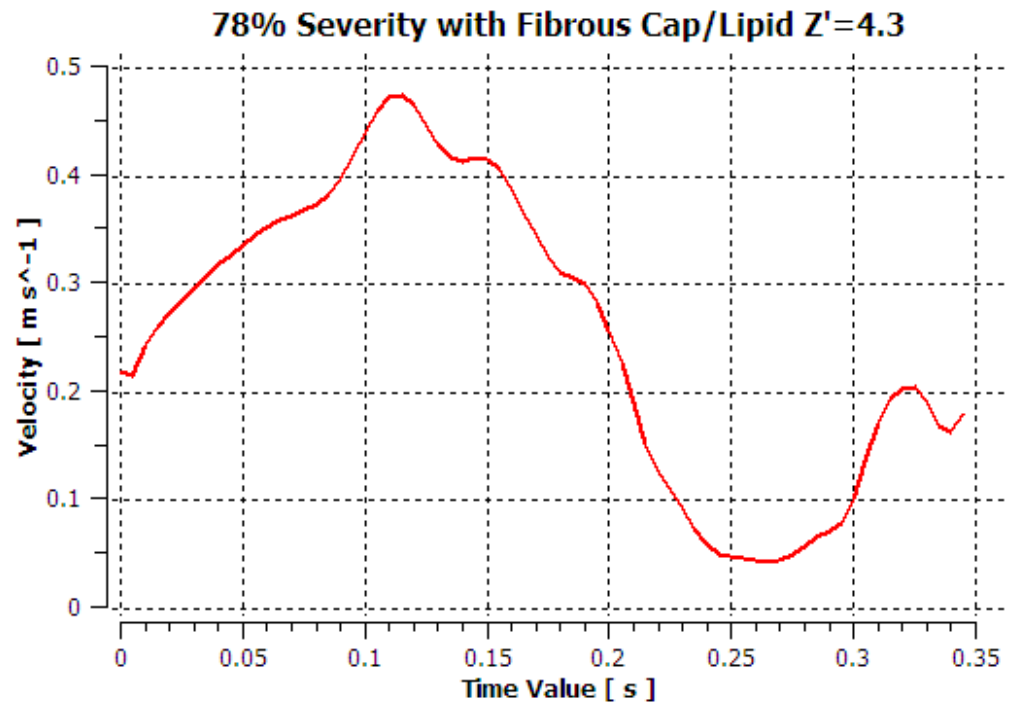

- Velocity



FiguRE 13: Velocity plots for $78 \%$ axis-asymmetric stenosed artery with multi-material properties. 

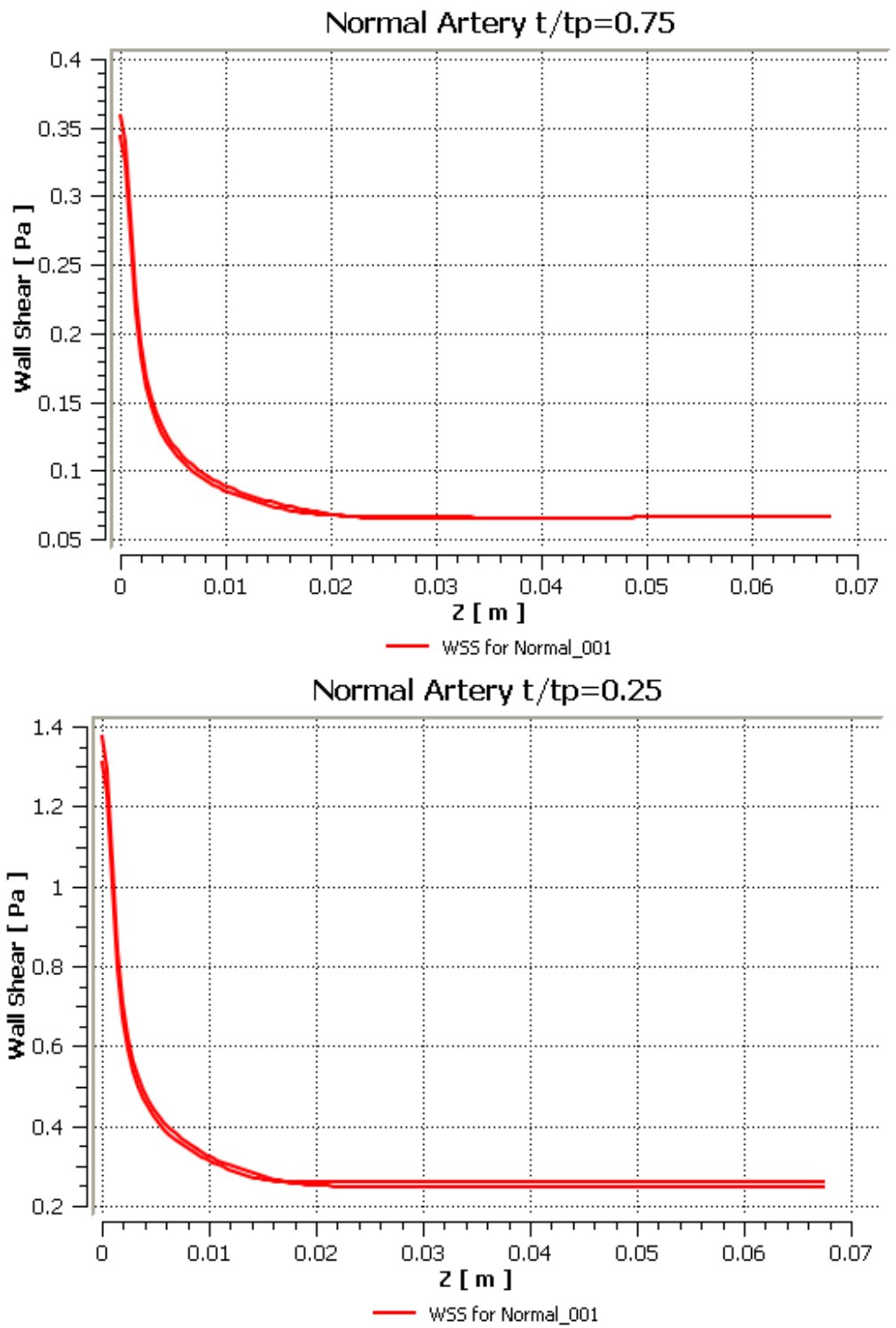

FiguRE 14: WSS at $\mathrm{t} / \mathrm{t} \mathrm{p}=0.25$ and $\mathrm{t} / \mathrm{t} \mathrm{p}=0.75$ for a normal artery. 
The $78 \%$ severity multi-material model with fibrous cap and lipid pool reveals more wss fluctuation (Figure 11) in the post stenotic region when compared to that of the uniform wall material model (Figure 9). Due to the increase in the thickness of the fibrous cap, the effect of the lipid pool is masked, resulting in more wss fluctuation in the post stenotic region. The wss magnitudes on the healthy side of the artery wall at the stenotic contraction are significantly smaller than the uniform material stenosis model. This trend is observed for all time steps. Thus, using the multi-material stenosis model is necessary for obtaining more accurate WSS values.

Pressure variations at all time steps (Figure 12) show almost identical trends to the uniform material stenosis model, when compared to that in Figure 9. This may be linked to the behaviour of the fibrous cap. With a thicker fibrous cap, pressure is not expected to vary as much as that of the $45 \%$ severity model.

Figure 13(a) shows that velocity variation at the post stenotic point $\left(Z^{\prime}=4.3\right)$ has a similar degree of distortion as the uniform material stenosis model (Figure 10(a)) of the same severity. This also suggests that the thick fibrous cap in combination with highly severe stenosis masks the effect of the soft lipid pool at the centre of the stenosis. The velocity vector plot (Figure 13(b)) reveals a less concentrated zero velocity region immediately after the stenosis. This suggests that although flow recirculation is still present, the stenosis allows more blood volume to pass through in certain areas, due to the increased flexibility of the stenosis.

To evaluate how many times greater the wss in a stenosed artery is than that in a normal artery of the same dimension, a ratio of the maximum wss to the wss at outlet of the same artery model is sufficient, providing the length of the artery model is adequate to satisfy the accuracy requirement of the numerical solution. In order to confirm the adequacy of the artery model length, a two way FSI simulation of the same Casson blood flow through a normal artery of the same dimension was carried out. Figure 14 presents the WSS results at two time steps, respectively. Comparing Figure 14 to Figure 8 
(a $78 \%$ stenosed artery at the same time steps), the normal artery model shows a constant WSS value from $z=0.0145$ to the end of the artery, whereas the stenosed artery shows a large level of wss distortion at the stenosis and in the post stenotic regions. However, the wsS values at the inlet and outlet of the normal artery correspond to that of the healthy side of the stenosed artery model. This confirms that the length of our stenosis artery model is adequate, thus the numerical results at the outlet of a stenosed artery represent that of a normal artery of the same dimension. The maximum wsS value is approximately eight times greater at the stenosis contraction than that in a normal artery for both time intervals.

\section{Conclusion}

In summary, the two way FSI solution of a non-Newtonian blood flow through a stenosed artery provides a more realistic simulation over the one way FSI solution. Moreover, the artery models used in the two way FSI simulation are further improved by constructing the stenosis with a fibrous cap and lipid pool regions and assigning different material properties to different regions. Consequently, FSI simulations of the multi-material stenosed model provide more reliable wss data for clinical analysis of the disease progression, as it shows less WSS at the stenotic region and minimises the fluctuations of wss and pressure in the post stenotic region, along with reduced flow recirculation zone near the stenosis.

Other features of the two way FSI simulation are:

1. the peak WSS is located at the stenotic contraction;

2. the maximal wss peak is found at $t / t p=0.25$ and minimal wss peak at $\mathrm{t} / \mathrm{tp}=0.75$

3. the pressure results show a mostly linear trend, apart from the stenotic contraction, where a small increase in pressure precedes a sharp drop. 


\section{References}

[1] K. C. Ang and J. N. Mazumdar, Mathematical modelling of three-dimensional flow through an asymmetric arterial stenosis, Mathematical and Computer Modelling, 25, No 1, 19-29, 1997, doi:10.1016/S0895-7177(96)00182-3. C587

[2] W. Y. Chan, Y. Ding, J. Y. Tu, Modeling of non-Newtonian blood flow through a stenosed artery incorporating fluid-structure interaction, ANZIAM Journal, 47, 507-523, 2007, http://anziamj.austms.org. au/ojs/index.php/ANZIAMJ/article/view/1059. C588, C590, C591

[3] Santabrata Chakravarty and Prashanta Kumar Mandal, Two-Dimensional blood flow through tapered arteries under stenotic conditions, Non-Linear Mechanics, 35, 779-793, August, 2006, doi:10.1016/S0020-7462(99)00059-1. C587

[4] Samuel A. Kock and Jens V. Nygaard. Nikolaj Eldrup,Ernst-Torben Frund and Anette Klaeke, William P.Pausk. Erling Falk and W.Yong Kim, Mechanical Stresses in carotid plaques using MRI-based fluid-structure interaction models, Journal of Biomechanics, 41, 1651-1658, March, 2008, doi:10.1016/j.jbiomech.2008.03.019. C590

[5] K. W. Lee and X. Y. Xu, Modelling of flow and wall behaviour in a mildly stenosed tube, Medical Engineering and Physics, 24, 575-586, May, 2002, doi:10.1016/S1350-4533(02)00048-6. C588

[6] M. X. Li and J. J. Beech-Brandt and L. R. John and P. R. Hoskins and W. J. Easson, Numerical analysis pulsatile blood flow and vessel wall mechanics in different degrees of stenoses, Journal of Biomechanics, 40, 3715-3724, June, 2007, doi:10.1016/j.jbiomech.2007.06.023. C590

[7] B. Pincombe and J. Mazumdar, The effects of post-stenotic dilatations on the flow of a blood analogue through stenosed coronary arteries, 
Mathematical and Computer Modelling, 25, No 6, 57-70, 1997, doi:10.1016/S0895-7177(97)00039-3. C587

[8] J. Poiseuille, Observations of blood flow, Ann. Sci. Naturelles Srie 5, 2, 1836. C587

[9] S. U. Siddiqui and N. K. Verma and Shailesh Mishra and R. S. Gupta, Mathematical modelling of pulsatile flow of Cassons fluid in arterial stenosis, Applied Mathematics and Computation, 2007, doi:10.1016/j.amc.2007.05.070. C587

[10] S. U. Siddiqui and Shailesh Mishra, A study of modified Casson's fluid in modelled normal and stenotic capillary-tissue diffusion phenomena, Applied Mathematics and Computation, 189, 1048-1057, 2007, doi:10.1016/j.amc.2006.11.151. C587

[11] Dalin Tang and Chun Yang and David N. Ku, A 3D thin-wall model with fluid-structure interactions for blood flow in carotid arteries with symmetric and asymmetric stenoses, Computers and Structures, $\mathbf{7 2}$, 357-377, 1999, doi:10.1016/S0045-7949(99)00019-X. C588

[12] Dalin Tang and Chun Yang and Yan Huang and David N. Ku, Wall stress and strain analysis using a three-dimensional thick-wall model with fluid-structure interactions for blood flow in carotid arteries with stenoses, Computers and Structures, 72, 341-356, May, 1999, doi:10.1016/S0045-7949(99)00009-7. C588

[13] Dalin Tang, Chun Yang, Jie Zheng, Pamela K. Woodard, Gregorio A. Sicard, Jeffrey E. Saffiz, Shunichi Kobayashi, Thomas K. Pilgram, and Chun Yuan, 3D computational mechanical analysis for human Atherosclerotic plaques using MRI-based models with fluid-structure interactions, MICCAI, 328-336, 2004. C588

[14] Dalin Tang and Chun Yang and Jie Zheng and Pamela K.Woodard and Gregorio A.Sicard and Jefferey E. Saffitz and Chun Yuan, 3D MRI-based 
multicomponent FSI models for artherosclerotic plaques, Biomedical Engineering, 32, 947-960, July, 2004. C588

\section{Author addresses}

1. C. X. Chen, School of Mathematical and Geospatial Sciences, RMIT University, Melbourne, Australia.

mailto: colin.chen@rmit. edu.au

2. Y. Ding, School of Mathematical and Geospatial Sciences, RMIT University, Melbourne, Australia. mailto:yan.ding@rmit.edu. au

3. J. A. Gear, School of Mathematical and Geospatial Sciences, RMIT University, Melbourne, Australia. mailto: jag@rmit.edu.au 